\title{
ON THE ABSOLUTE CONVERGENCE OF A FOURIER-HERMITE EXPANSION OF NONLINEAR FUNCTIONALS
}

\section{MARGARET OWCHAR AND ARNOLD J. TINGLEY 1}

1. Introduction. R. H. Cameron and C. Hatfield $[1 ; 2]$ have studied the convergence of orthogonal developments of nonlinear functionals defined on the Wiener space $C$. In this paper their results will be extended.

The set of functionals $\left\{\psi_{m_{1}}, \cdots, m_{N}(x)\right\} \quad\left(m_{i}=0,1,2, \cdots ; N\right.$ $=1,2, \cdots)$ has been shown [3] to be a C.O.N. set in the space $C$ where $\psi_{m_{1}}, \cdots, m_{N}(x)=\prod_{i=1}^{N} H_{m_{i}}\left(\int_{0}^{1} \alpha_{i}(t) d x(t)\right), H_{j}(u)$ being the partially normalized Hermite polynomial $(-1)^{j} 2^{-j / 2}(j !)^{-1 / 2} e^{u^{2}} d^{j} e^{-u^{2}} / d u^{j}$, and $\left\{\alpha_{j}(t)\right\}$ being any C.O.N. set of real-valued functions on $[0,1]$ such that each $\alpha_{j}(t)$ is of bounded variation. If the integrals appearing in the definition of the functionals $\psi_{m_{1}}, \ldots, m_{N}(x)$ are interpreted as the generalized Riemann-Stieltjes integrals of Paley, Wiener, and Zygmund [6], it is not necessary to assume that each $\alpha_{j}(t)$ is of B.V., but this interpretation ,will not be necessary in this paper, as we shall always understand that $\alpha_{j}(t)=2^{1 / 2} \cos ((2 j-1) 2) \pi t$. by

The Fourier-Hermite coefficients of a functional $F(x)$ are defined

$$
A_{m_{1}, \cdots, m_{N}}=\int_{c}^{w} F(x) \psi_{m_{1}, \cdots, m_{N}}(x) d_{w} x
$$

where these integrals exist. (As we confine our attention to functionals of class $L_{2}(C), A_{m_{1}}, \cdots, m_{N}$ will necessarily exist for each set of subscripts.)

Cameron and Hatfield proved the following theorem:

TheOREM. Let $F(x)$ be of class $L_{2}(C)$, be continuous in the Hilbert topology at $x_{0} \in C$, and satisfy

$$
|F(x)| \leqq A \exp \left\{B \int_{0}^{1}[x(t)]^{2} d t\right\}
$$

where $A$ and $B$ are non-negative constants. For each positive integer $N$ let

Received by the editors May 4, 1953.

1 The work done by Tingley on this paper was supported in part by the Office of Ordnance Research of the U. S. Army. 


$$
\sum_{m_{1}, \cdots, m_{N}=0}^{\infty} A_{m_{1}, \cdots, m_{N}} \psi_{m_{1}}, \ldots, m_{N}\left(x_{0}\right)
$$

converge absolutely, where $A_{m_{1}}, \ldots, m_{N}$ is defined as in (1). Then

$$
F\left(x_{0}\right)=\lim _{N \rightarrow \infty} \sum_{m_{1}, \cdots, m_{N}=0}^{\infty} A_{m_{1}, \cdots, m_{N}} \psi_{m_{1}}, \ldots, m_{N}\left(x_{0}\right) .
$$

A recent paper [5] by one of the authors has suggested sufficient conditions for the absolute convergence of (3). These are given in the theorem of the following section.

Ross E. Graves [4] also has found sufficient conditions for the convergence of Fourier-Hermite developments, which, however, differ substantially from ours. It is, in fact, readily seen from examples that in some respects his conditions are weaker, and in other respects stronger, than those of the following theorem.

2. Conditions for the convergence of a Fourier-Hermite development.

Theorem. Let $F(x)$ be of class $L_{2}(C)$, be continuous in the Hilbert topology on $C$, and satisfy

$$
|F(x)| \leqq A \exp \left\{B \int_{0}^{1}[x(t)]^{2} d t\right\}
$$

where $A$ and $B$ are non-negative constants. Let $m_{1}, \cdots, m_{N}$ be any set of non-negative integers, and let $i_{k}$ be the integer $i$ satisfying

$$
\sum_{j=1}^{i-1} m_{j}<k \leqq \sum_{j=1}^{i} m_{j}
$$

Define

$$
y_{i}(t)=2^{1 / 2} \int_{0}^{t} \cos \frac{(2 i-1)}{2} \pi s d s,
$$

and for each $k \leqq P=\sum_{i=1}^{N} m_{i}$ let the kth variation of $F(x)$ with respect to $y_{i_{e_{1}}}, y_{i_{s_{2}}}, \cdots, y_{i_{\alpha_{k}}}$, where $s_{1}, \cdots, s_{k}$ are any distinct integers chosen from $1,2, \cdots, P$, exist, be continuous in the uniform topology on $C$, and for some number $\eta>0$ satisfy

$$
\begin{aligned}
\sup _{|n| \leqq \eta}\left|\delta^{(k)} F\left(x+h y_{i_{s_{k}}} \mid y_{i_{s_{1}}}, \cdots, x_{i_{s_{k}}}\right)\right| & \\
& \cdot \prod_{j=1}^{P-k}\left(1+\left|\int_{0}^{1} y_{i_{r_{j}}}^{\prime}(t) d x(t)\right|\right) \in L_{1}(C),
\end{aligned}
$$


$r_{1}, r_{2}, \cdots, r_{P-k}$ being those of the first $P$ positive integers not included in $s_{1}, \cdots, s_{k}$. For some $\delta>0$ let

$$
\begin{aligned}
\left|\delta^{(2 v)} F\left(x \mid y_{q_{1}}, y_{q_{1}}, y_{q_{2}}, y_{q_{2}}, \cdots, y_{q_{\nu}}, y_{q_{\nu}}\right)\right| \\
\cdot \exp \left\{\sum_{i=1}^{N}\left(\frac{1+\delta}{2}\right)\left[\int_{0}^{1} y_{i}^{\prime}(t) d x(t)\right]^{2}\right\} \in L_{1}(C)
\end{aligned}
$$

for each $\nu \leqq N$, and each choice of $\left\{q_{j}\right\}$ where $q_{1}<q_{2}<\cdots<q_{\nu}$ are distinct integers chosen from $1,2, \cdots, N$. Then it follows that for each $x_{0} \in C$, the Fourier-Hermite development of $F(x)$ converges in the sense that the equality (4) holds.

PROOF. It is clear that we need only show that, under the hypothesis of our theorem, the series (3) converges absolutely.

Making use of the special case of Theorem 1 of [5] we have

$$
A_{m_{1}, \cdots, m_{N}}=\prod_{j=1}^{N}\left\{2^{-m_{j} / 2}\left(m_{j} !\right)^{-1 / 2}\right\} \int_{c}^{w} \delta^{(P)} F\left(x \mid y_{i_{1}}, y_{i_{2}}, \cdots, y_{i_{P}}\right) d_{w} x,
$$

(5) and (6) ensuring that the assumptions of that theorem are satisfied. Now by the assumed continuity of the $k$ th variation of $F(x)$, for each $k$, the $P$ variations of $F(x)$ may be taken in any order. For $m_{i}>2$, each $i$, we take first the $2 N$ variations with respect to $y_{1}, y_{1}, y_{2}, y_{2}, \cdots, y_{N}, y_{N}$, and then the $P-2 N$ variations with respect to the remaining $y$ 's. Thus,

$$
\begin{aligned}
A_{m_{1}}, \cdots, m_{N}= & \prod_{j=1}^{N}\left\{2^{-m_{j} / 2}\left(m_{j} !\right)^{-1 / 2}\right\} \\
& \cdot \int_{c}^{w} \delta^{(P-2 N)} G\left(x \mid y_{i_{8}}, \cdots, y_{i_{m_{1}}}, y_{i_{m_{1}}+2}, \cdots, y_{i_{P}}\right) d_{w} x,
\end{aligned}
$$

where $G(x)=\delta^{(2 N)} F\left(x \mid y_{1}, y_{1}, \cdots, y_{N}, y_{N}\right)$.

Conditions (6) and (7) ensure that $G(x)$ and the set $y_{i_{3}}, \cdots$, $y_{i_{m_{1}}}, y_{i_{m_{1}}+3}, \cdots, y_{i_{P}}$ satisfy the assumptions of the theorem referred to above. Hence,

$$
A_{m_{1}}, \cdots, m_{N}=\prod_{j=1}^{N}\left\{\frac{1}{2\left(m_{j}\left(m_{j}-1\right)\right)^{1 / 2}}\right\} \int_{c}^{w} G(x) \psi_{m_{1}-2, \cdots, m_{N-2}}(x) d_{w} x .
$$

It is known that ${ }^{2}$

2 The corresponding formula for differently normalized polynomials is given in J. V. Uspensky's On the development of arbitrary functions in series of Hermite's and Laguerre's polynomials, Ann. of Math. vol. 28, p. 596, and is credited there to Adamoff's On asymptotic expressions of the polynomials $U_{n}(x)$, Annales Inst. Polytechnique de St. Petersburg vol. 5 (1906). 


$$
\max _{-\infty<u<\infty}\left|H_{n}(u) e^{-((1+\delta) / 2) u^{2}}\right| \leqq K\left(\frac{1}{n}\right)^{1 / 4}
$$

where $\delta$ is a fixed positive number, and $K$ is a constant depending on $\delta$.

Using (8) and (9), we have, for any $x_{0} \in C$,

$$
\begin{aligned}
\sum_{m_{1}, \cdots, m_{N}=3}^{\infty} & \left|A_{m_{1}, \cdots, m_{N}} \psi_{m_{1}, \cdots, m_{N}}\left(x_{0}\right)\right| \\
\leqq & K^{2 N} \sum_{m_{1}, \cdots, m_{N}=3}^{\infty} \prod_{j=1}^{N}\left[\frac{1}{2\left(m_{j}\left(m_{j}-1\right)\right)^{1 / 2}\left(m_{j}\left(m_{j}-2\right)\right)^{1 / 4}}\right. \\
& \left.\cdot \exp \left\{\frac{1+\delta}{2}\left(\int_{0}^{1} y_{j}^{\prime}(t) d x_{0}(t)\right)^{2}\right\}\right] \\
& \cdot \int_{c}^{w}|G(x)| \exp \left\{\sum_{i=1}^{N} \frac{1+\delta}{2}\left[\int_{0}^{1} y_{i}^{\prime}(t) d x(t)\right]^{2}\right\} d_{w} x .
\end{aligned}
$$

Noting (7), it is clear that the right side of (10) is finite, so the left side is finite.

For at least one (but not all) of the $m_{j}$ 's fixed, and the remainder greater than 2, we get absolute convergence of the resulting series in a similar way. There are only a finite number of these series, and there are only a finite number of terms of (3) for which all $m_{j}$ are less than 3 . Thus the theorem is proved.

\section{BIBLIOGRAPHY}

1. R. H. Cameron and C. Hatfield, On the summability of certain developments of nonlinear functionals, Bull. Amer. Math. Soc. vol. 55 (1949) pp. 130-145.

2. - On the summability of certain series for unbounded nonlinear functionals, Proc. Amer. Math. Soc. vol. 4 (1953) pp. 377-387.

3. R. H. Cameron and W. T. Martin, The orthogonal development of non-linear functionals in series of Fourier-Hermite functionals, Ann. of Math. vol. 48 (1947) pp. 385-392.

4. Ross E. Graves, Functionals of finite degree and the convergence of their FourierHermite developments, Proc. Amer. Math. Soc. vol. 4 (1953) pp. 95-101.

5. Margaret Owchar, Wiener integrals of multiple variations, Proc. Amer. Math. Soc. vol. 3 (1952) pp. 459-470.

6. R. E. A. C. Paley, N. Wiener, and A. Zygmund, Notes on random functions, Math. Zeit. vol. 37 (1933) pp. 647-668.

Manitoba Cancer Relief and Research Institute and UNIVERSITY OF NEbRASKa 